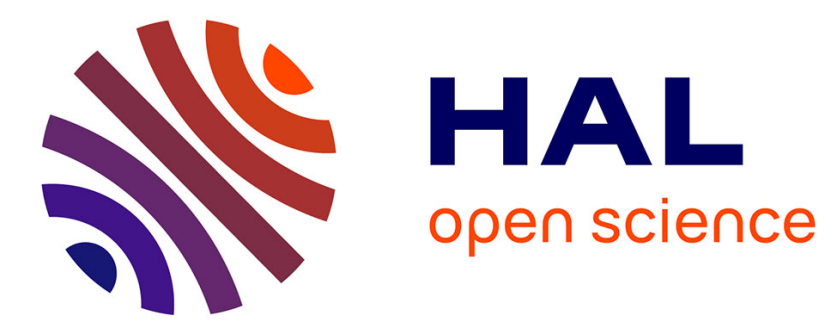

\title{
Garder ou tomber la blouse ?
}

Franck Burellier, Annick Valette

\section{To cite this version:}

Franck Burellier, Annick Valette. Garder ou tomber la blouse ?. Journal de gestion et d'économie médicales, 2011, 29 (6), pp.336-345. 10.3917/jgem.116.0336 . hal-02272393

\section{HAL Id: hal-02272393 \\ https://hal.science/hal-02272393}

Submitted on 27 Aug 2019

HAL is a multi-disciplinary open access archive for the deposit and dissemination of scientific research documents, whether they are published or not. The documents may come from teaching and research institutions in France or abroad, or from public or private research centers.
L'archive ouverte pluridisciplinaire HAL, est destinée au dépôt et à la diffusion de documents scientifiques de niveau recherche, publiés ou non, émanant des établissements d'enseignement et de recherche français ou étrangers, des laboratoires publics ou privés. 


\title{
Garder ou tomber la blouse?
}

Analyse de la transition vers un rôle de médecin-gestionnaire au sein de deux établissements hospitaliers français

\author{
Franck Burellier \\ Université Lyon III / IAE de Lyon / Centre de recherche Magellan \\ Université de Grenoble / CERAG \\ franck.burellier@univ-lyon3.fr
}

Annick Valette

Université de Grenoble / Faculté d'économie / CERAG

annick.valette@upmf-grenoble.fr 


\title{
RESUME
}

En tentant de déléguer la réflexion médico-économique aux médecins, la création des pôles d'activité constitue un enjeu clé de la Nouvelle Gouvernance Hospitalière. Nous nous intéressons ici à la transition de médecins vers le rôle de responsable de pôle d'activité, rôle hybride associant clinique et gestion. Souvent décrite comme insurmontable par la littérature, nous questionnons cette transition et la capacité d'ajustement des médecins : comment des médecins s'ajustent-ils à un rôle de médecin-gestionnaire? Nous répondons à cette question par une étude de cas menée dans deux hôpitaux, un $\mathrm{CH}$ et un CHU. A partir d'une grille d'analyse des postures d'ajustement, nos résultats montrent que si la souplesse de l'organisation permet effectivement à certains médecins de définir leur propre conception du rôle et d'échapper à la prescription forte, d'autres au contraire mènent une profonde transition individuelle.

\section{MOTS-CLES}

Transition de rôle - Ajustement à un rôle - Médecins-gestionnaires - Hôpital

\begin{abstract}
Aiming at delegating economic responsibilities to physicians, the creation of clinical directorates is a key issue of hospitals' new organization. In this research, we focus on the transition from a regular physician role to a clinical director role - an hybrid role mixing medical and management missions. Though litterature describes this transition as difficult, we question it, as well as doctors' ability of adjustment : how do doctors adjust to a doctor-manager role ? We answer this question with a case study we lead in two hospitals, a classic hospital and a teaching one. Thanks to a model of analysis of adjustment situations, our results show that, though organizational flexibility enables some doctors to redefine their own role concept and to escape from high prescriptions, other doctors however do a deep individual transition.
\end{abstract}

\section{KEYWORDS}

Role transition - Role adjustement - Doctors-managers - Hospitals 


\section{INTRODUCTION}

"[Ca veut dire quoi pour vous être chef de pôle?] C'est pas nous qui faisons la loi. Le cadre d'exercice des responsabilités est défini réglementairement. Donc, je suis quelqu'un de pragmatique : il y a une règle, on la joue, et on la joue du mieux qu'on perçoit. D’autant que si cette règle vous définit à grands traits dans des textes, c'est à nous de la faire vivre et de l'interpréter. »

Le responsable du pôle Couple-Enfant (CHU)

Influencé par le New Public Management, et dans un contexte de fort déficit, le secteur hospitalier français est engagé depuis la dernière décennie dans un ensemble de réformes visant à améliorer sa performance (Every, 2005). La réforme que nous étudions ici est celle de la Nouvelle Gouvernance Hospitalière (2007) et plus précisément son volet relatif à la création du rôle de chef de pôle d'activité. Les pôles, constitués à partir de regroupements de services, sont des entités de mutualisation des ressources et de déconcentration de la gestion. Ils sont dirigés par des médecins, dont les missions vont au-delà des responsabilités antérieurement confiées aux chefs de service. Ces missions s'apparentent à des objectifs : contractualisation avec la direction autour d'objectifs et de moyens médico-économiques, ingénierie de projets transversaux, organisation du pôle. Mais elles renvoient aussi à des méthodes de travail : animation de réunions, recrutement des collaborateurs, management du personnel du pôle.

Cette réforme exige ainsi des médecins nommés chefs de pôle qu'ils réalisent une transition vers un rôle de médecin-gestionnaire (Ferlie et al., 1996). Or, une littérature abondante tirée des expériences étrangères souligne les difficultés qu'il y a à tenir un rôle hybride de professionnel-gestionnaire (voir notamment pour les médecins : Ashburner et al., 1996 ; Button et Roberts, 1997 ; Fitzgerald et Ferlie, 2000 ; Kirkpatrick et Ackroyd, 2003 ; Llewellyn, 2001 ; Loubet, 2007). Elle montre en particulier que, les professionnels tendent souvent à détourner les objectifs de ce nouveau rôle en affaiblissant la dimension gestionnaire pour conserver intacte la dimension professionnelle (voir notamment Dechamp et Romeyer, 2006 ; McGivern et al., 2006). Ces changements touchent en effet des individus dont les compétences et l'identité sont fortement normées par leur profession d'appartenance (Abbott, 1988 ; Freidson, 1988).

Il nous apparait ainsi intéressant d'étudier le cas français et d'interroger ce type de transition : comment des médecins s'ajustent-ils à un rôle de médecin-gestionnaire ? Nous répondons à cette question par une étude de cas menée dans deux hôpitaux, un $\mathrm{CH}$ et un $\mathrm{CHU}$. Nous mobilisons une grille d'analyse des postures d'ajustement proposée par Nicholson (1984). Nos résultats montrent que si la souplesse de 
l'organisation permet effectivement à certains médecins de définir leur propre conception du rôle et d'échapper à la prescription forte, d'autres au contraire mènent une profonde transition individuelle.

\section{L'INDIVIDU FACE AU ROLE PRESCRIT : QUI S'AJUSTE ?}

Nous nous intéressons au phénomène de transition de rôle. Nous l'envisageons comme un mouvement psychologique, voire physique, qu'effectue un individu lorsqu'il passe d'une position à une autre (Ashforth, 2001). Alors que la littérature sur les transitions vers des rôles de professionnel-gestionnaire met l'accent sur les difficultés à tenir le rôle-prescrit et conclut à la toute puissance du professionnel,, une littérature plus générale sur les transitions de rôle envisage ce phénomène comme un processus souple, permettant des combinaisons d'ajustements entre l'individu et le rôle prescrit (voir notamment Ashforth et Sakes, 1995 ; Munton et West, 1995 ; Neal et Griffin, 2006 ; Nicholson, 1984 ; West et Rushton, 1989). Une transition de rôle peut à la fois exiger certaines adaptations de la part de son occupant, mais aussi laisser de l'espace à ce dernier pour ajuster le rôle à ses besoins.

Dans cette logique, nous avons choisi de mobiliser la typologie de Nicholson (1984), qui se fonde sur l'idée qu'il n'y a pas systématiquement concordance entre les attentes liées au nouveau rôle et l'individu censé l'occuper. La transition consiste alors en des processus d'interactions, ou d'ajustements, entre le rôle prescrit et son occupant. Nicholson définit quatre types d'ajustements, formulés du point de vue de l'individu : la réplication, l'absorption, la détermination, et l'exploration.

La réplication correspond à une absence d'ajustement. Il n'y a ni changement dans les caractéristiques personnelles, ni modification des composants du rôle. L'individu prend le rôle sans chercher à le transformer. L'absorption se réfère à une situation d'ajustement de la part de l'individu seulement. Le rôle n'est pas, ou peu altéré. L'individu s'adapte aux attentes du rôle, quitte à faire évoluer fortement ce qu'il est. La détermination se rapporte à une situation d'ajustement par le rôle uniquement. L'individu ne s'adapte pas, ou peu, personnellement. Il produit au contraire un effort pour transformer le rôle tandis que lui-même conserve son cadre de référence. Il façonne son rôle en fonction de ce qu'il est. Enfin, l'exploration représente un ajustement mutuel de l'individu et du rôle. Le rôle transforme l'individu en même temps que l'individu fait évoluer le rôle. Il peut s'agir soit d'une situation intermédiaire (où individu et rôle ont trouvé un compromis d'ajustement), soit d'une situation totalement inédite. 
UNE ETUDE DE LA TRANSITION VERS LE ROLE DE RESPONSABLE DE POLE DANS DEUX HOPITAUX

Nous avons opté pour une étude de cas qualitative. Les deux cas choisis sont un Centre Hospitalier Universitaire (CHU) et un Centre Hospitalier $(\mathrm{CH})$. Le CHU étudié dispose de 2000 lits et compte environ 9000 personnels dont 1500 médecins. Au moment de notre étude, il connait un déficit de près de 6,5 millions d'euros. La mise en place des pôles y a débuté en 2006 par une phase préparatoire qualifiée de «pré-pôles ». Les pôles d'activités cliniques et médico-techniques y sont au nombre de 18. Chacun d'eux est dirigé par un médecin (PU-PH ou MCU-PH), assisté par un cadre soignant et un cadre administratif, et supervisé par un directeur référent. La gestion de la transition a été légère, autant au niveau de la nomination (critères de sélection peu explicites) que de l'accompagnement (se limitant à la présentation de la réforme et la création d'une fiche de poste).

Moins grand que le CHU, le CH étudié compte 1000 lits et environ 2000 agents dont un peu plus de 200 médecins. Au moment de notre étude, l'établissement est également en déficit (nous n'avons pas pu accéder aux données). L'instauration des pôles s'y est faite en 2007, et ne semble avoir été qu'une formalité car la quasi-totalité des pôles d'activité créés a repris les mêmes contours que les structures préexistantes. Les pôles cliniques et médico-techniques y sont au nombre de 8 . Chacun d'eux est dirigé par un médecin $(\mathrm{PH})$ assisté par un cadre soignant, et supervisé par un directeur délégué. Pour des raisons financières et de taille des structures, il n'existe pas de cadres administratifs. A l'instar du CHU, la gestion de la transition a été légère, qu'il s'agisse de la nomination (critères de sélections obscurs) ou de l'accompagnement (avec aussi une présentation de la réforme, mais absence de fiche de poste).

Notre investigation a été menée à partir d'entretiens individuels semi-directifs et de recherches documentaires. De septembre 2008 à mars 2010, nous avons réalisé 34 entretiens, dont 22 au sein du CHU (14 responsables de pôle et 8 membres de la direction) et 12 au sein du $\mathrm{CH}$ (8 responsables de pôle et 4 membres de la direction). Ces entretiens ont porté sur des thématiques permettant d'approcher le travail effectif réalisé par les responsables de pôle (les missions, les activités quotidiennes, les interactions, les évolutions du rôle) ; ainsi que leur personnalité (l'accompagnement, leurs intérêts, leur évolution dans le rôle, leurs valeurs personnelles). En parallèle, nous avons recueillis des documents internes aux deux hôpitaux (fiches de poste, contrats de pôle, présentations...) et externes (circulaires, 
code de la santé publique...). Ces documents nous ont permis de mieux cerner les attentes institutionnelles (instances publiques) et locales (établissements étudiés) concernant le rôle de responsable de pôle.

Pour analyser nos matériaux, nous avons procédé à une analyse de contenu thématique manuelle, en suivant la grille d'analyse élaborée par Nicholson (1984). Nous avons classé sous la dimension «ajustement par l'individu» tous changements effectués par les médecins dans leurs compétences, identité, valeurs, et personnalité. Par exemple, un discours mettant l'accent sur l'apprentissage de nouvelles compétences disciplinaires a été catégorisé comme un fort ajustement par l'individu.

«Si vous voulez, [devenir responsable de pôle] ça m’a décentré de mes métiers d'origine, hein. Parce que les gens auxquels je m'intéresse maintenant font de la rééducation, ils font dans le cardiaque... c'est quand même des métiers que je ne connais pas bien. Donc j'ai été obligé d'apprendre. »

Le responsable du pole Rééducation et Physiologie (CHU)

Nous avons classé sous la dimension « ajustement par le rôle » toutes les variations mises en œuvre dans le travail prescrit par l'organisation, qu'il s'agisse des objectifs ou des méthodes de travail à employer pour y parvenir. Par exemple, un discours indiquant la délégation de la réflexion médico-économique a été classé comme un fort ajustement par le rôle.

«Mes défauts c'est que je vais pas toujours à fond dans mes dossiers, les trucs comme ça. J'aime bien déléguer les trucs qui m'emmerdent. Je suis pas un homme de dossiers, hein. Je suis un homme d'idées. ^

Le responsable du pôle Digidune (CHU)

Ces deux dimensions nous ont permis de catégoriser les responsables de pôle (voir l'annexe 1).

LE MEDECIN FACE AU ROLE DE MEDECIN-GESTIONNAIRE PRESCRIT : DES AJUSTEMENTS INDIVIDUELS POSSIBLES SANS DIFFICULTES

A l'issu de l'analyse, la répartition des 22 responsables de pôle se fait de manière homogène dans les postures de réplication, d'absorption, et de détermination. Seule l'exploration fait figure d'exception (voir la figure I). 
Ajustement par l'individu

Faible

Fort

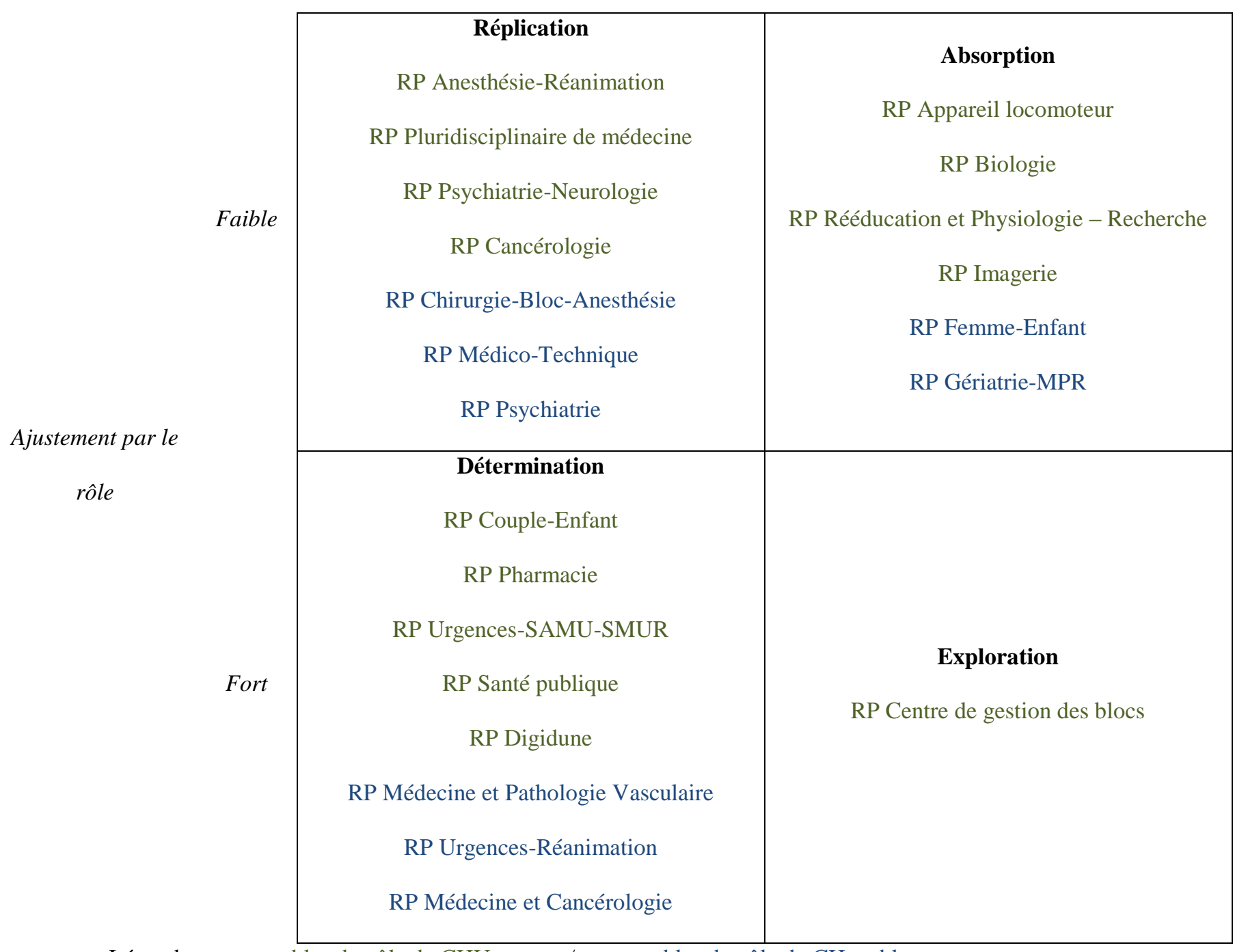

Légende : responsables de pôle du CHU en vert / responsables de pôle du CH en bleu

Figure I. Les postures d'ajustement des 22 responsables de pôle du CHU et du CH

La posture de réplication peut ici être résumée par l'idée : « je savais déjà faire, je faisais déjà comme ça avant, donc je continue à faire la même chose ». Les médecins dans cette posture ont effectué la transition de rôle car ils n'avaient, en fait, aucun ajustement à réaliser, ni personnel, ni dans leur manière de faire. En tant que chefs de service, ils fonctionnaient déjà comme des responsables de pôle : attention particulière au coût des soins, mise en place de projets transversaux... 
«Vous voyez, il y a 15 ans, je publiais sur « coût-efficacité de l'EPO dans les interventions ». C'est pas nouveau. Je veux dire, faut pas croire que c'est arrivé comme ça. Il y a des gens qui disent «plus on avance, plus on retarde ». Moi je suis dans une discipline où, depuis longtemps, comme on coûtait les plus chers, on a dî s'y intéresser, hein. Et donc on a travaillé avec l'économie de la santé. »

Le responsable du pôle Santé Publique (CHU)

Cela ne veut cependant pas dire que l'individu ne rencontre aucune difficulté à mener cette transition. En effet, parmi les sept membres de cette catégorie, deux se trouvent dans une situation inconfortable de conflit de rôle (les responsables des pôles Cancérologie et Anesthésie-Réanimation du CHU). Le premier (le responsable du pôle Cancérologie), est confronté à un conflit de rôle lié à l'élargissement du périmètre de gestion, alors que le second (la responsable du pôle Anesthésie-Réanimation) se trouve face à un conflit dû à la prise de distance par rapport au terrain. Toutefois, dans ces deux cas, les réactions aux conflits sont différentes : l'un souhaite quitter sa fonction, malgré les refus successifs de la direction (le responsable du pôle Cancérologie); l'autre continue à le tenir (la responsable du pôle AnesthésieRéanimation). Dans les deux cas cependant, les difficultés n'ont pas d'incidence sur la tenue effective du rôle. Les objectifs sont respectés.

La posture d'absorption peut ici être interprétée comme : «je ne sais pas faire, mais je veux m'adapter». Les médecins en absorption ont tous mené la transition car ils apprennent leur rôle en l'expérimentant, par le développement de nouvelles compétences de gestion (5 cas sur 6), et exceptionnellement par l'intégration de nouvelles valeurs médico-économiques ( 1 cas sur 6 , le responsable du pôle GériatrieMPR du CH).

«Je me surprends de temps en temps à dire « on ne peut pas faire parce que financièrement on ne peut pas ». Et pourtant ce n'était vraiment pas mon truc. L'histoire des chambres tampons, pour reprendre ça, c'est que ça combine les deux : l'économique et le clinique. Ça ne me gêne pas d'avoir des chambres tampons pour le bien des malades. Et ça permet de faire des économies. »

Le responsable du pôle Gériatrie-MPR $(\mathrm{CH})$

La charge de l'apprentissage pour l'individu est relativement faible car son ajustement se fait au travers de son travail quotidien et par l'interaction avec ses cadres assistants et son directeur délégué/référent. 
Tous les médecins dans cette posture avaient un attrait préexistant pour la gestion, ou l'ont développé en prenant le rôle. Ils n'ont donc pas vécu cet ajustement personnel comme difficile.

La situation de détermination correspond ici à l'idée : «je ne sais pas ou ne veux pas faire et je ne veux pas m'adapter ». Les médecins n’ont pas effectué la transition de rôle car aucun d'eux n'a souhaité le tenir, ou apprendre à le tenir. Cet ajustement ne signifie cependant pas que ces médecins ne comprennent pas la logique du rôle. Ils préfèrent simplement ne pas la mettre en application dans leur activité, soit par désintérêt personnel pour la gestion, soit par priorisation des missions cliniques. Ils ajustent alors ce qu'ils font à ce qu'ils sont.

Les modifications observées concernent aussi bien les objectifs du rôle (5 cas sur 8), comme l'optimisation des ressources utilisées ou la collaboration interdisciplinaire, que les méthodes de travail (3 cas sur 8), comme l'organisation de réunions ou la rédaction du contrat de pôle. Dans tous les cas, ces deux types de détermination sont indépendants : certains médecins, bien que n'adoptant pas la logique médico-économique, continuent à mettre en place des réunions de pôle afin de discuter des problématiques de terrain, de la même façon que certains d'entre eux adoptent une logique médicoéconomique, mais abandonnent les activités bureaucratiques. Le coût pour l'individu est nul car rien ne change dans son travail.

«Moi je suis suffisamment sur le terrain... J'y suis tout le temps. Je dis pas que je travaille tout le temps, mais je passe le matin, je passe le midi, je passe le samedi, je passe le dimanche. Les gens ils savent que je suis là. Mais moi je dis, je suis là pour le patient. Le reste, c'est pas mon problème. Non, mais, le reste, c'est du satellite. Il y a un patient, c'est centré sur le patient. »

La responsable du pôle Urgences-SAMU-SMUR (CHU)

Enfin, la posture d'exploration peut ici être résumée par l'idée : «je ne sais pas faire, mais je veux m'adapter et en faire plus ». Le seul médecin compris dans cette catégorie a non seulement effectué la transition, car il a appris son rôle, mais également fait évoluer celui-ci, en prenant des initiatives allant audelà ce qui était attendu. Cette combinaison, plus rare, semble se produire lorsqu'un médecin s'intéresse personnellement à la gestion, et est prêt à s'adapter personnellement si besoin, en apprenant de nouvelles compétences de gestion. 
"[Le DG] trouvait que c'était bien de faire un système qui gère les blocs transversalement, et ainsi de suite. Et moi, à l'époque, alors que j'étais pressenti pour le [pôle] Tête et Cou, j'avais déjà amendé dans ce sens-là, en disant "c'est une excellente idée, il faut faire ça! Il faut faire un Centre de Gestion des

Blocs! ». Un truc transversal qui soit prestataire de services et qui mette à disposition des chirurgiens les salles, les personnels, les matériels... C'est lourd, mais c'est un truc qui fait un gain de productivité fantastique!»

Le responsable du pôle Centre de Gestion des Blocs (CHU)

A priori très bénéfique pour l'organisation car permettant d'innover, cette posture est cependant risquée. La preuve en est que le médecin en question a été obligé de démissionner de son rôle de responsable pour ne pas remettre trop brutalement en question la culture de l'établissement. L'individu doit non seulement s'investir dans un apprentissage continu (des prescriptions et de ses initiatives), mais aussi se confronter à des personnes opposées à son action (ici, les autres chirurgiens).

\section{LE MEDECIN, MAITRE DE SA TRANSITION ?}

Il n'existe donc pas une forme d'ajustement typique observé sur nos cas dans la transition vers un rôle de médecin-gestionnaire. En particulier, l'argument répandu dans la littérature d'une transition systématiquement difficile pour tenir un rôle de médecin-gestionnaire n'apparaît pas ici. En effet, sur 22 médecins étudiés, seuls 5 n’ont pas du tout réussi la transition (ils ont redéfini les objectifs du rôle), et 3 ne l'ont réussi que partiellement (ils ont détourné certaines activités). La transition vers le rôle de médecin-gestionnaire est donc possible, par réplication ou absorption du rôle, et éventuellement par détermination lorsque celle-ci concerne seulement les méthodes de travail. La résistance à la transition de rôle n'apparaît que lorsque les objectifs du rôle sont remis en question ou lorsque le rôle imaginé brusque la culture organisationnelle.

Ces différentes situations représentent une charge variable pour l'organisation. La réplication en constitue la posture la plus bénéfique car elle n'exige aucun accompagnement. L'absorption lui est également avantageuse car l'individu se forme seul sur le terrain. La détermination est en revanche la situation la plus coûteuse car elle exige de l'organisation une prise en charge pour combler les manques. Cette intervention ne concerne pas directement les responsables de pôle mais leurs collaborateurs (cadres 
assistants ou directeurs référents, par exemple), auxquels la direction demande soit de l'aide sur certaines activités, soit une suppléance totale au responsable de pôle. Enfin, l'exploration, en raison de son éventuelle contestation, peut exiger pour l'organisation le remplacement du médecin désigné.

Les facteurs influençant les postures d'ajustement sont certainement multiples et en interaction. Nous souhaiterions mettre en avant ici le poids de la spécialité médicale, le rôle de gestion par l'établissement, mais surtout les caractéristiques individuelles des médecins. Certaines spécialités médicales, parce qu'elles ont une tradition de contraintes économiques fortes ont depuis longtemps socialisé les médecins à la gestion et favorisent les ajustements individuels (par exemple, en Anesthésie-Réanimation). A contrario, d'autres spécialités aux prises avec des contraintes cliniques fortes, font apparaitre les enjeux de gestion économiques comme secondaires dans la gestion du temps et favorisent la conservation du rôle en l'état (par exemple, en Urgences). L'accompagnement quand il est plus formalisé, comme c'est le cas au CHU, pourrait faciliter les ajustements individuels. Nous notons toutefois que la gestion de ces transitions (recrutement, accompagnement, formation), est légère et laisse alors place au poids des caractéristiques individuelles dans le processus d'ajustement. Ainsi, le parcours professionnel (par exemple la présidence de la CME), les activités extra-professionnelles (par exemple, les mandats d'élus municipaux), l'intérêt pour la gestion, ou encore les systèmes de valeurs peuvent expliquer la plus ou moins grande souplesse des individus à faire évoluer le rôle joué.

Notre travail comporte certaines limites. Une première limite concerne notre méthode de recueil des matériaux. Nous avons majoritairement procédé à des entretiens. Notre analyse se base donc seulement sur le discours de nos interlocuteurs. Or, une meilleure compréhension du rôle de responsable de pôle mériterait une appréciation plus directe des faits, que seule une méthode d'observation permettrait d'étudier. Une deuxième limite est relative à la grille d'analyse que nous mobilisons et à notre méthode de codage. Nous considérons que la théorie proposée par Nicholson (1984), bien qu'utile pour catégoriser des situations-types, reste trop fermée. Les individus ont parfois des postures ambigües, où des changements s'effectuent à la fois dans leur rôle et dans ce qu'ils sont (sur certaines caractéristiques, et pas sur d'autres). Une analyse plus inductive des ajustements permettrait de comprendre la complexité des situations rencontrées par les médecins. Une troisième limite est également liée à notre méthodologie, mais de manière plus globale. Par contrainte, nous n'avons pu étudier le phénomène de transition que de manière instantanée, en effectuant une photographie des postures d'ajustement des médecins quelques 
mois après leur prise de fonction. Or, une transition correspond à un processus. Cet aspect processuel n'a pas pu être envisagé dans ce travail. L'étude des ajustements effectués par les médecins de manière longitudinale pourrait constituer une autre voie de recherche.

\section{CONCLUSION}

Cet article avait pour objectif d'étudier le phénomène de transition de médecins vers des rôles de responsables de pôle. Présentés comme piliers de la Nouvelle Gouvernance Hospitalière, ces rôles associant activités cliniques et gestionnaires ne vont cependant pas de soi. En effet, la littérature met l'accent sur les difficultés rencontrées par les médecins pour effectuer la transition vers ces rôles. Nous avons alors questionné la capacité d'ajustement des médecins face à ce type de rôle : comment des médecins s'ajustent-ils à un rôle de médecin-gestionnaire ? Nous avons répondu à cette question par une étude de cas menée dans deux hôpitaux, un $\mathrm{CH}$ et un CHU. A partir d'une grille d'analyse des postures d'ajustement, nos résultats ont montré que si la souplesse de l'organisation permet effectivement à certains médecins de définir leur propre conception du rôle et d'échapper à la prescription forte, d'autres au contraire mènent une profonde transition individuelle. Notre discussion a souligné le peu d'influence des caractéristiques disciplinaires et organisationnelles sur ces transitions, à l'inverse des profils individuels des médecins.

\section{REFERENCES}

Abbott A.D. (1988), The system of professions: essay on the division of expert labour, Chicago, University of Chicago Press.

Ashburner L., Ferlie E., et FitzGerald L. (1996), Organizational transformation and top-down change: the case of the NHS, British Journal of Management, vol. 7, ${ }^{\circ} 1$, p. 1-16.

Ashforth B.E. (2001), Role transitions in organizational life: an identity-based perspective, Mahwah, New Jersey, Lawrence Erlbaum Associates.

Ashforth B.E. et Sakes A.M. (1995), Work role transitions: a longitudinal examination of the Nicholson model, Journal of Occupational and Organizational Psychology, vol. 68, p. 157-175. 
Button W. J. et Roberts G. (1997), Communication, clinical directorates, and the corporate NHS, Journal of Public Relations Research, vol. 9, n², p. 141-162.

Dechamp G. et Romeyer C. (2006), Trajectoires d'appropriation des principes de nouvelle gouvernance hospitalière par les médecins, $15^{\text {ème }}$ Congrès de l'AIMS, Annecy.

Ferlie E., Ashburner L., Fitzgerald L., et Pettigrew A.M. (1996), The new public management in action, Oxford, Oxford University Press.

Fitzgerald L. et Ferlie E. (2000), Professionals: back to the future?, Human Relations, vol. 53, n ${ }^{\circ}$, p. 713-739.

Freidson E. (1988), Profession of medicine: a study of the sociology of applied knowledge, Chicago, University of Chicago Press.

Kirkpatrick I. et Ackroyd S. (2003), Archetype theory and the changing professional organization: a critique and alternative, Organization, vol. 10, n 4 , p. 731-749.

Llewellyn S. (2001), “Two-way window”: clinicians as medical managers, Organization Studies, vol. 22, $\mathrm{n}^{\circ} 4$, p. 593-623.

Loubet G. (2007), Du praticien hospitalier au médecin-gestionnaire : hybridation du métier et tensions de rôle. Une étude exploratoire, Doctorat de sciences de gestion, Université Montpellier II.

McGivern G., Addicott R., Ferlie E., et FitzGerald L. (2006), "Incidental" and "strategic"clinicalmanagerial hybrid identity, $22^{\text {nd }}$ EGOS colloquium, Bergen.

Munton A. et West M. (1995), Innovation and personal change: patterns of adjustment to relocation, Journal of Organizational Behavior, vol. 16, n4, p. 363-374.

Neale M. et Griffin M. A. (2006), A model of self-held work roles and role transitions, Human Performance, vol. $19, \mathrm{n}^{\circ} 1$, p. 23-41.

Nicholson N. (1984), A theory of work role transitions, Administrative Science Quarterly, vol. 29, p. 172191.

West M.A. (1987), Role innovation in the world of work, British Journal of Social Psychology, vol. 26, $n^{\circ} 4$, p. 305-315. 


\begin{tabular}{|c|c|c|c|}
\hline Posture & Responsable de pôle (RP) & Ajustement par le rôle & Ajustement par l'individu \\
\hline $\begin{array}{l}\text { Posture de } \\
\text { réplication }\end{array}$ & $\begin{array}{l}\text { RP Anesthésie Réanimation } \\
\text { (CHU) } \\
\text { RP Pluridisciplinaire de } \\
\text { médecine (CHU) } \\
\text { RP Psychiatrie-Neurologie } \\
\text { (CHU) } \\
\text { RP Cancérologie (CHU) } \\
\text { RP Chirurgie-Bloc- } \\
\text { Anesthésie }(\mathrm{CH}) \\
\text { RP Médico-Technique }(\mathrm{CH})\end{array}$ & $\begin{array}{l}\text { Aucun : respect volontaire ou } \\
\text { contraint des missions } \\
\text { prescrites } \\
\text { - contractualisation } \\
\text { - réflexion médico- } \\
\text { économique } \\
\text { - ingénierie de projets } \\
\text { transversaux } \\
\text { - organisation du pôle } \\
\text { - animation de réunions } \\
\text { - recrutement } \\
\text { - management du personnel }\end{array}$ & $\begin{array}{l}\text { Aucun : adéquation préalable } \\
\text { des compétences et valeurs } \\
\text { personnelles, sans évolution } \\
\text { notable depuis la prise de } \\
\text { fonction }\end{array}$ \\
\hline \multirow{4}{*}{$\begin{array}{l}\text { Posture } \\
\text { d'absorption }\end{array}$} & $\begin{array}{l}\text { RP Appareil locomoteur } \\
\text { (CHU) }\end{array}$ & $\begin{array}{l}\text { Faible : respect des missions } \\
\text { prescrites, légère réduction du } \\
\text { nombre de réunions prescrites }\end{array}$ & $\begin{array}{l}\text { Fort : apprentissage du travail } \\
\text { collectif et de la délégation }\end{array}$ \\
\hline & $\begin{array}{l}\text { RP Biologie (CHU) } \\
\text { RP Rééducation et } \\
\text { Physiologie }(\mathrm{CHU})\end{array}$ & $\begin{array}{l}\text { Aucun : respect des missions } \\
\text { prescrites }\end{array}$ & $\begin{array}{l}\text { Fort : apprentissage de } \\
\text { spécialités médicales et du } \\
\text { management }\end{array}$ \\
\hline & $\begin{array}{l}\text { RP Imagerie }(\mathrm{CHU}) \\
\text { RP Femme-Enfant }(\mathrm{CH})\end{array}$ & $\begin{array}{l}\text { Aucun : respect des missions } \\
\text { prescrites }\end{array}$ & $\begin{array}{l}\text { Fort : apprentissage } \\
\text { managérial }\end{array}$ \\
\hline & RP Gériatrie-MPR (CH) & $\begin{array}{l}\text { Aucun : respect des missions } \\
\text { prescrites }\end{array}$ & $\begin{array}{l}\text { Fort : léger apprentissage de la } \\
\text { gestion comptable, évolution } \\
\text { vers des valeurs médico- } \\
\text { économiques }\end{array}$ \\
\hline \multirow{4}{*}{$\begin{array}{l}\text { Posture de } \\
\text { détermination }\end{array}$} & $\begin{array}{l}\text { RP Couple-Enfant (CHU) } \\
\text { RP Pharmacie (CHU) } \\
\text { RP Urgences-SAMU-SMUR } \\
\text { (CHU) } \\
\text { RP Médecine et Cancérologie } \\
\text { (CH) }\end{array}$ & $\begin{array}{l}\text { Fort : refus d'endosser la } \\
\text { réflexion médico-économique, } \\
\text { focalisation sur les missions } \\
\text { cliniques }\end{array}$ & $\begin{array}{l}\text { Aucun : préservation des } \\
\text { compétences antérieures, } \\
\text { revendication de valeurs } \\
\text { cliniques préexistantes }\end{array}$ \\
\hline & RP Santé publique (CHU) & $\begin{array}{l}\text { Fort : contournement des } \\
\text { lignes hiérarchiques, refus de } \\
\text { signer les contrats de pôle }\end{array}$ & $\begin{array}{l}\text { Aucun : préservation des } \\
\text { compétences antérieures, } \\
\text { revendication de valeurs } \\
\text { médico-économiques }\end{array}$ \\
\hline & RP Digidune (CHU) & $\begin{array}{l}\text { Fort : respect des objectifs } \\
\text { prescrits, mais délégation des } \\
\text { activités médico-économiques }\end{array}$ & $\begin{array}{l}\text { Faible : léger apprentissage } \\
\text { managérial, revendication de } \\
\text { valeurs cliniques préexistantes }\end{array}$ \\
\hline & $\begin{array}{l}\text { RP Médecine et Pathologie } \\
\text { Vasculaire }(\mathrm{CH}) \\
\text { RP Urgences-Réanimation } \\
(\mathrm{CH})\end{array}$ & $\begin{array}{l}\text { Fort : refus d'endosser la } \\
\text { réflexion médico-économique, } \\
\text { réduction du nombre de } \\
\text { réunions prescrites }\end{array}$ & $\begin{array}{l}\text { Aucun : préservation des } \\
\text { compétences antérieures, } \\
\text { revendication de valeurs } \\
\text { cliniques préexistantes }\end{array}$ \\
\hline $\begin{array}{l}\text { Posture } \\
\text { d'exploration }\end{array}$ & $\begin{array}{l}\text { RP Centre de gestion des } \\
\text { blocs (CHU) }\end{array}$ & $\begin{array}{l}\text { Fort : participation à la } \\
\text { création du pôle, prises } \\
\text { d'initiatives dans la conduite } \\
\text { du changement }\end{array}$ & $\begin{array}{l}\text { Fort : apprentissage de } \\
\text { compétences en gestion, } \\
\text { préservation de valeurs de } \\
\text { prise de recul préexistantes }\end{array}$ \\
\hline
\end{tabular}

Annexe 1. Justification des postures d'ajustement des 22 responsables de pôle du CHU et du CH 